\title{
Ultrafast control of magnetic interactions via light-driven phonons
}

\author{
D. Afanasiev $\oplus^{1,7} \llbracket$, J. R. Hortensius ${ }^{1,7}$, B. A. Ivanov ${ }^{2,3}$, A. Sasani ${ }^{4}{ }^{4}$, E. Bousquet ${ }^{\circledR}{ }^{4}$, Y. M. Blanter ${ }^{1}$, \\ R. V. Mikhaylovskiy ${ }^{5}{ }^{5}$ A. V. Kimel ${ }^{6}$ and A. D. Caviglia ${ }^{1 \otimes}$
}

\begin{abstract}
Resonant ultrafast excitation of infrared-active phonons is a powerful technique with which to control the electronic properties of materials that leads to remarkable phenomena such as the light-induced enhancement of superconductivity $^{1,2}$, switching of ferroelectric polarization ${ }^{3,4}$ and ultrafast insulator-to-metal transitions ${ }^{5}$. Here, we show that light-driven phonons can be utilized to coherently manipulate macroscopic magnetic states. Intense mid-infrared electric field pulses tuned to resonance with a phonon mode of the archetypical antiferromagnet $\mathrm{DyFeO}_{3}$ induce ultrafast and long-living changes of the fundamental exchange interaction between rare-earth orbitals and transition metal spins. Non-thermal lattice control of the magnetic exchange, which defines the stability of the macroscopic magnetic state, allows us to perform picosecond coherent switching between competing antiferromagnetic and weakly ferromagnetic spin orders. Our discovery emphasizes the potential of resonant phonon excitation for the manipulation of ferroic order on ultrafast timescales ${ }^{6}$.
\end{abstract}

The ability to control macroscopic states of matter by light on the fastest possible timescale crucially relies on finding efficient routes to manipulate the various microscopic interactions that define the stability of the cooperative state ${ }^{7}$. In magnetism, these interactions either involve the electrons of only one magnetic ion, for example, single-ion magnetic anisotropy ${ }^{8,9}$, or rely on a mutual interaction between pairs of magnetic ions, for example, exchange coupling ${ }^{10}$. Despite the intrinsic differences, both of these interactions originate from the same electrostatic Coulomb repulsion, strongly sensitive to the lattice symmetry and the electronic overlap. Terahertz control of magnetism is a thriving field, but its main focus has been, until now, limited either to the direct excitation of spins by the magnetic field component of the single-cycle terahertz pulse ${ }^{11}$ or to resonant pumping of the electronic degrees of freedom ${ }^{9,12}$. Resonant pumping of infrared-active phonons with multiterahertz pulses in the mid-infrared (MIR) spectral range has emerged as a low-energy route to drive large-amplitude coherent net structural distortions. These lattice distortions, which emerge on the timescale of several picoseconds, modulate the spatial overlap of the electronic wavefunctions and have been shown to transiently melt charge ${ }^{13}$ and orbital ordering ${ }^{14}$, drive metal-insulator phase transitions ${ }^{5}$ and even enhance superconducting correlations in high-temperature cuprate superconductors ${ }^{1,2}$. Although pioneering experiments demonstrated that driving optical phonons can also affect magnetism ${ }^{15-18}$, no coherent switching of the spin orientation or coherent light-induced magnetic symmetry breaking has been shown so far. Here we investigate phonon-induced magnetism (phonomagnetism) in dysprosium orthoferrite $\left(\mathrm{DyFeO}_{3}\right)$, a material in which a strong exchange interaction between the spin of the transition metal (TM) ion and the orbital momentum of the rare-earth (RE) ion leads to a distinctive first-order spin-reorientation phase transition accompanied by a change of the magnetic symmetry from the antiferromagnetic (AFM) to the weakly ferromagnetic (WFM) ${ }^{19}$. We show that a subpicosecond pulse of an intense multiterahertz electric field, tuned in resonance with a phonon mode (Fig. 1a), drives a coherent spin reorientation, which develops a long-living WFM order within a half-cycle of the spin precession. Phonon-induced magnetism emerges via a non-equilibrium metastable state (Fig. 1b), inaccessible not only via a thermodynamic transformation but also via optical pumping of the high-energy electronic transitions. We experimentally and theoretically demonstrate that phonomagnetism originates from phonon-induced lattice distortions that lead to ultrafast modification of the RE-TM exchange interaction.

The magnetic insulator $\mathrm{DyFeO}_{3}$ crystallizes in a perovskite orthorhombic structure and exhibits AFM spin order set by the $\mathrm{Fe}-\mathrm{Fe}$ isotropic exchange interaction. Although the singlet state of the $\mathrm{Fe}^{3+}$ ion $\left({ }^{6} \mathrm{~S}\right.$ ground state) results in negligible single-ion magnetic anisotropy, its anisotropic exchange interaction with the large angular momentum of the dysprosium ion $\left({ }^{6} \mathrm{H}_{15 / 2}\right.$ ground state) mediates the strong coupling of the iron spins to the crystal lattice, and thereby sets up the magnetocrystalline anisotropy ${ }^{19}$. The thermal population of the two lowest $\mathrm{Dy}^{3+}$ Kramers doublets (energy gap $\Delta E=6.4 \mathrm{meV}$ ) changes the orbital state of the RE. This change defines the strength and symmetry of the Fe-Dy exchange coupling, which leads to a spontaneous first-order spin reorientation transition. During this transition, at the Morin temperature $T_{\mathrm{M}}=51 \mathrm{~K}$, the direction of the magnetic easy axis for the iron spins changes abruptly between the $y$ - and $x$-crystal axis. The reorientation is accompanied by a change in magnetic symmetry, from a collinear AFM to a canted WFM state, characterized by the emergence of a net magnetization $M$.

The spin reorientation in $\mathrm{DyFeO}_{3}$ is described by the magnetic potential $F$, which depends on the temperature and the angle $\varphi$ the spins form with the $y$-axis (Supplementary Note 2). In a broad temperature range this potential function features two characteristic minima at 0 and $90^{\circ}$ (Fig. $1 \mathrm{~b}$ ), which signals two ordered states. The height of the potential barrier that separates the two competing

${ }^{1}$ Kavli Institute of Nanoscience, Delft University of Technology, Delft, the Netherlands. ${ }^{2}$ Institute of Magnetism, National Academy of Sciences and Ministry of Education and Science, Kiev, Ukraine. ${ }^{3}$ National University of Science and Technology MISiS, Moscow, Russian Federation. ${ }^{4}$ CESAM QMAT Physique Théorique des Matériaux, Université de Liège, Liège, Belgium. ${ }^{5}$ Department of Physics, Lancaster University, Bailrigg, UK. ${ }^{6}$ Institute for Molecules and Materials, Radboud University Nijmegen, Nijmegen, the Netherlands. ${ }^{7}$ These authors contributed equally: D. Afanasiev, J. R. Hortensius.

凶e-mail: dmytro.afanasiev@physik.uni-regensburg.de; a.caviglia@tudelft.nl 
a

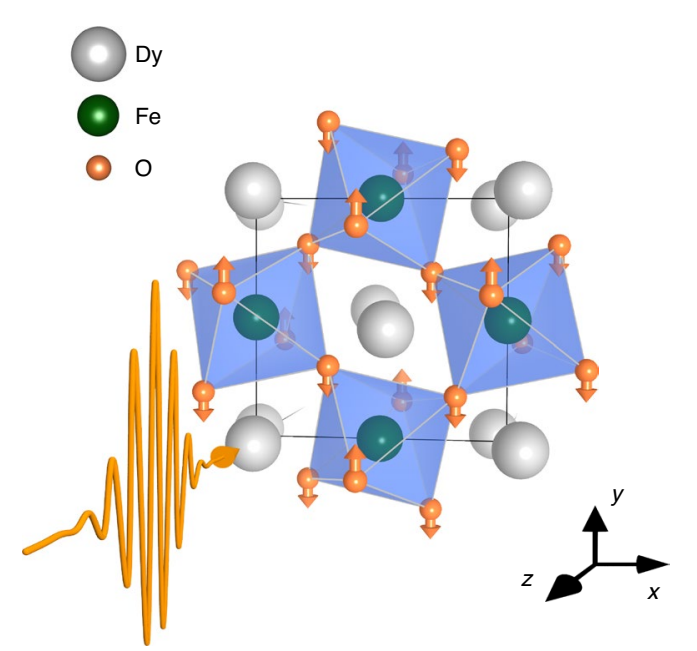

b

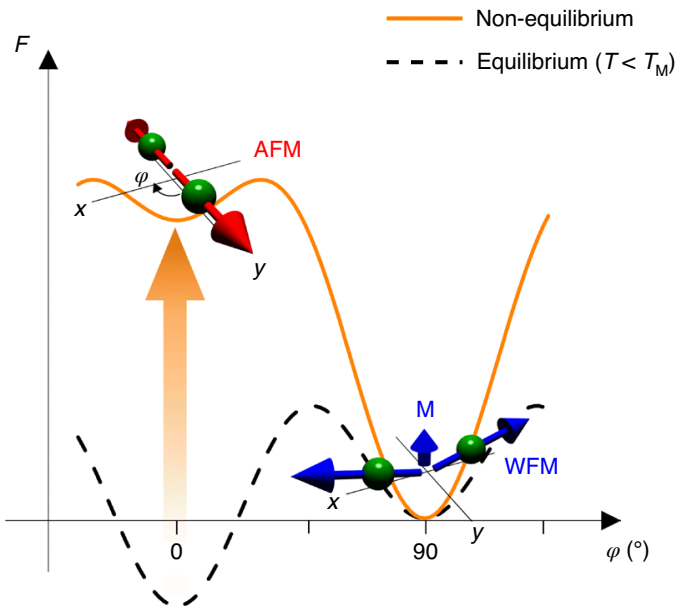

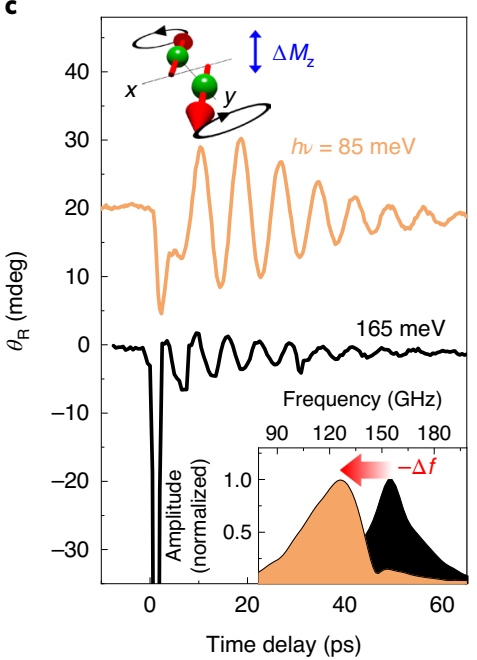

d

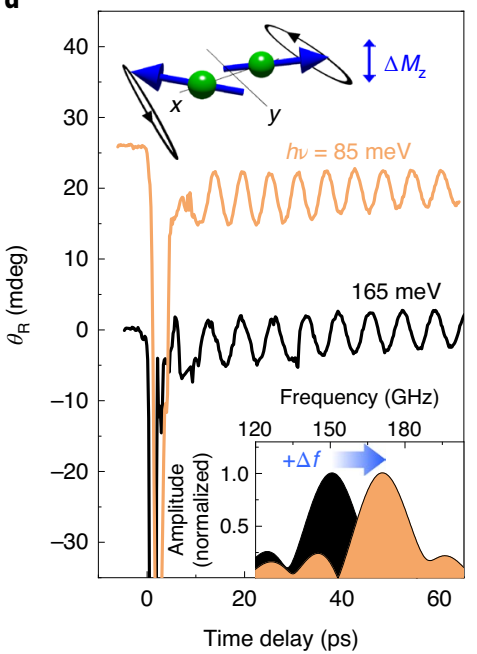

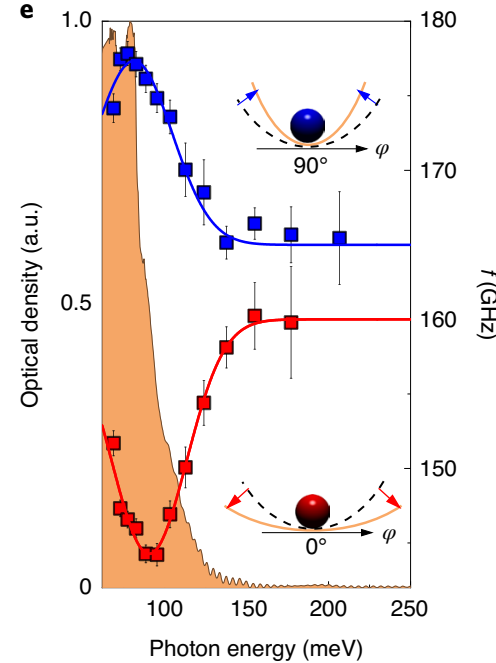

Fig. 1 | Phonon-driven reconfiguration of the magnetic potential in $\mathrm{DyFeO}_{3}$. $\mathbf{a}$, The eigenmode of the laser-excited $\mathrm{B}_{u}$ phonon mode; orange arrows indicate the atomic motion of the oxygen ions. $\mathbf{b}$. The magnetic potential $F$ as a function of the angle $\varphi$ that the spins form with the $y$ axis before (dashed black line) and after (orange line) phonon excitation. The potential is shown only for $T<T_{\mathrm{M}}$. The red and blue arrows depict the spin configurations that correspond to the antiferromagnetic (AFM) and weakly ferromagnetic(WFM) phases. The orange arrow highlights the destabilization of the AFM ground state together with the reduction of the potential barrier that separates the phases. $\mathbf{c} \mathbf{d}$, Time-resolved transient rotation of the probe polarization plane $\theta_{\mathrm{R}}$ after light excitation with photon energies of $85 \mathrm{meV}$ (orange traces) and $165 \mathrm{meV}$ (black traces) performed in the AFM (b) and WFM (c) phases. Bottom insets: normalized amplitude spectra of the soft mode oscillations. Top insets: schematics of the corresponding spin precessions, with the resulting oscillating magnetic component $\Delta M_{z}$. e, Central frequency of the excited soft mode as a function of the photon energy of the pump pulse in the two magnetic phases. The solid lines serve as a guide to the eye. The shaded curve shows the sample's optical density. Insets: schematics that indicate changes in the local curvature of the magnetic potential. a.u., arbitrary units. The error bars represent the standard error of the extracted frequency $f$.

states, as well as their relative energy, is controlled by the strength of the Fe-Dy exchange. This interaction is not only sensitive to the temperature, but also to changes in the crystal environment, both via direct modulation of the Fe-Dy electronic overlap and via structurally driven changes in the orbital state of the RE $4 f$ multiplet ${ }^{9,20}$. Here, we considered light-induced oscillations of atoms driven far from their equilibrium positions to control the strength of the $\mathrm{Fe}-$ Dy exchange $e^{21,22}$ and to realize lattice control of the spin arrangement on an ultrafast timescale.

In our experiments, we investigated spin dynamics in single crystals of $z$-cut $\mathrm{DyFeO}_{3}$ (Methods). The phonon excitation was provided by an intense (electric field in excess of $10 \mathrm{MV} \mathrm{cm}^{-1}$ ), phase-locked mid-infrared impulsive source ( $250 \mathrm{fs}, 1 \mathrm{kHz})$, tunable in a broad photon energy range from 65 to $250 \mathrm{meV}(16-60 \mathrm{THz})$. This energy range covers the highest frequency infrared-active transverse optical (TO) phonon mode $\left(\mathrm{B}_{\mathrm{u}}\right)$ centred at $70 \mathrm{meV}$ and associated with periodic stretching of the $\mathrm{Fe}-\mathrm{O}$ bonds (Fig. $\mathrm{Ia}$ and Supplementary Figs. 2 and 9). The ensuing spin dynamics were measured, in a conventional pump-probe scheme, by tracking the polarization rotation $\theta_{\mathrm{R}}$, imprinted by the magneto-optical Faraday effect, on co-propagating near-infrared probe pulses at the photon energy of $1.55 \mathrm{eV}$. In our experimental geometry, the Faraday rotation probes the magnetization dynamics along the normal direction. Importantly, the frequency $f$ of the antiferromagnetic spin precession mode (the soft mode of the Morin phase transition), informs us on the local curvature of the magnetic potential, according to the relation:

$$
\left.f^{2} \propto \frac{\partial^{2} F}{\partial \varphi^{2}}\right|_{\varphi=\varphi_{0}}
$$


both in the AFM $\left(\varphi_{0}=0^{\circ}\right)$ and $\operatorname{WFM}\left(\varphi_{0}=90^{\circ}\right)$ phases, similarly to Hase et al. ${ }^{23}$ and Yamaguchi et al. ${ }^{24}$ (also see Supplementary Note 3 ).

Figure 1c,d shows light-induced dynamics of the Faraday signal in the AFM and WFM phases and reveals coherent oscillations, which correspond to spin precession around the respective equilibria. These dynamics were induced by pump pulses tuned in resonance with the lattice vibrational mode $(85 \mathrm{meV})$. As a control experiment, we excited magnon oscillations via impulsive stimulated Raman scattering ${ }^{25}$ using pulses tuned away from lattice or electronic resonances $(165 \mathrm{meV})$. We note that the frequencies of the magnon oscillations excited by impulsive stimulated Raman scattering coincide exactly with the tabulated values reported in the literature ${ }^{24,26}$ and therefore serve as in situ probe of the curvature of the potential $F$ at equilibrium.

A comparison of the Fourier spectra (insets in Fig. 1c,d) reveals that the frequency of the spin precession excited by pulses in resonance with the lattice mode is shifted as compared to the equilibrium value. The sign of the shift $\Delta f$ depends on the initial magnetic configuration, being red in the AFM phase and blue in the WFM phase. To underline the resonant character of the frequency shift, we tuned the photon energy of the pump pulse across the phonon resonance and extracted the central frequency of the spin oscillations (Fig. 1e). The data acquired in both magnetic phases show that the onset of the frequency shift follows closely the spectrum of the linear absorption of the $\mathrm{B}_{\mathrm{u}}$ phonon mode and therefore reveals a correlation between the light-driven phonon and spin dynamics. These observations contain important information on the effects of the phonon pumping on the magnetic potential. The red shift indicates a flattening of the potential energy in the vicinity of the AFM minimum $\left(\varphi=0^{\circ}\right)$, which may lead to a phase instability. The blue shift observed in the WFM phase points at an increased curvature of the potential and enhanced phase stability in the vicinity of the WFM minimum $\left(\varphi=90^{\circ}\right)$.

A time-resolved Fourier analysis indicated that the oscillations were chirped and the change in frequency occurred within a half-cycle of the first spin oscillation, which thereby defines an upper bound for the phonon-driven changes in the magnetic potential of about 5 ps (Fig. 2a,b). This time is an order of magnitude shorter than that reported in Yamaguchi et al. ${ }^{24}$, in which the heat-driven dynamics of the magnetic potential were governed by the interaction of incoherent acoustic phonons with the $\mathrm{Dy}^{3+}$ electrons. Thus, the observed ultrafast response excludes heat-driven spin-lattice relaxation as the origin of the initial kinetics of the potential. Remarkably, the change in frequency persists for a time $\tau>100 \mathrm{ps}$, which exceeds the precession period by nearly an order of magnitude. We also note that the absolute value of $\tau$ closely follows the characteristic equilibrium decay time of the spin precession (Fig. 2b inset).

As shown in Fig. 3a, measurements of the frequency of the magnetic mode at equilibrium as a function of temperature revealed a cusp-like evolution in proximity to $T_{\mathrm{M}}$, in excellent agreement with Balbashov et al. ${ }^{26}$. In contrast, the frequency of the magnons launched via resonant phonon excitation was characterized by a split up of the magnon branches at $T_{\mathrm{M}}$, which led to a pronounced discontinuity of more than $50 \mathrm{GHz}$ (Fig. 3a). The discontinuity leads to the emergence of magnetic responses at frequencies well below the minimum value attainable at equilibrium $(140 \mathrm{GHz})$. Although the heat capacity of $\mathrm{DyFeO}_{3}$ changes by a factor of 20 between 10 and $60 \mathrm{~K}$ (ref. ${ }^{27}$ ), the magnitude of the observed frequency change is nearly temperature independent in the range $10-100 \mathrm{~K}$, which provides another indication that a non-thermal process is at play. From the analysis of the temperature dependence of the magnon frequencies, we mapped out the magnetic potential $F$ (Supplementary Note 3$)$ before $(t<0)$ and after $(t>0)$ laser excitation (Fig. 3b). Importantly, phonon pumping substantially increases the energy of the AFM state, which simultaneously lowers the potential barrier,
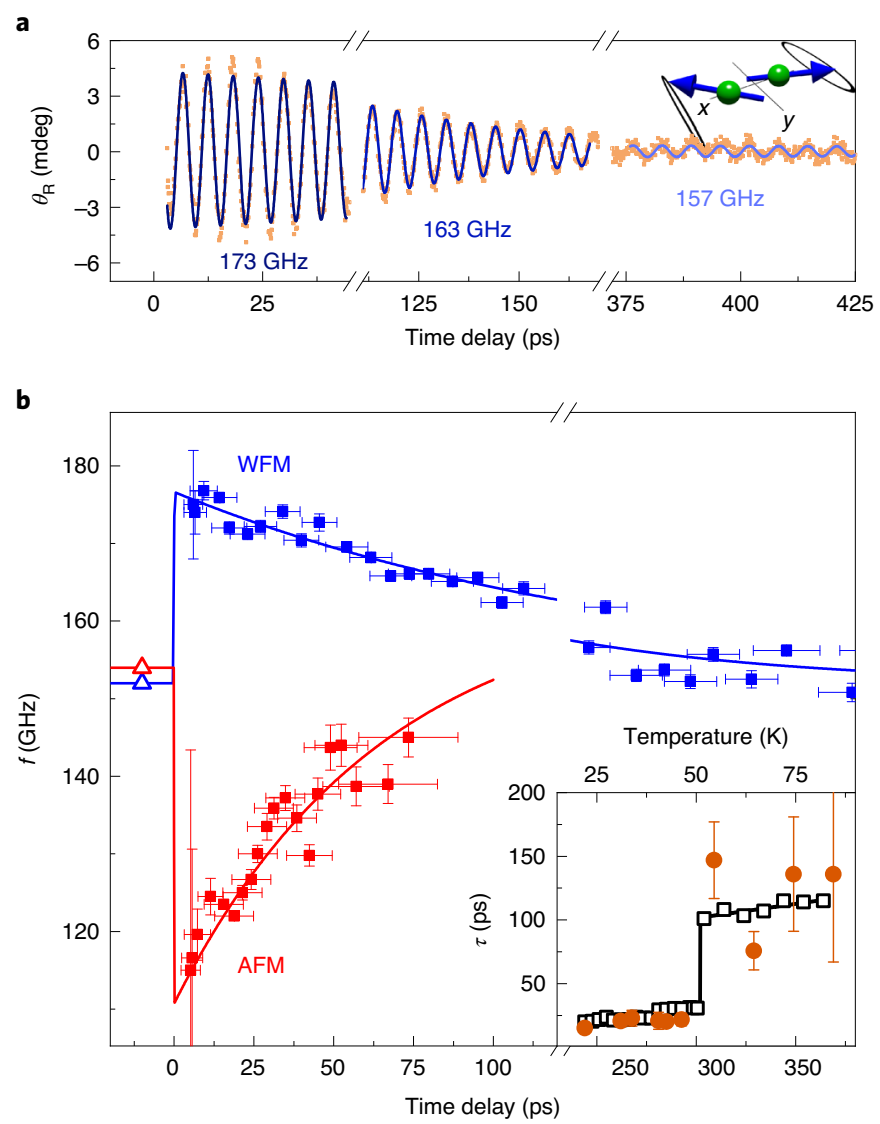

Fig. 2 | Ultrafast dynamics of the soft mode frequency. a, Trace of the spin precession dynamics after resonant phonon excitation $(h \nu=85 \mathrm{meV})$ in the WFM phase. The solid blue lines are sine fits to extract a time-varying frequency $f$. $\mathbf{b}$, The resulting dynamics of the frequency $f$ as measured in the AFM (red, $T=43 \mathrm{~K}$ ) and WFM (blue, $T=57 \mathrm{~K}$ ) phases. The solid lines are guides to the eye. The triangular markers at $t<0$ correspond to reference measurements performed at a pump photon energy of $165 \mathrm{meV}$. The horizontal error bars indicate the time window of the sine fit. The vertical error bars represent the standard error of the extracted frequency $f$. Inset: relaxation time $\tau$ of the frequency across $T_{M}$ (orange circles) imposed on the decay time of the spin precession (black squares). The inset error bars represent the standard error of the extracted relaxation time $\tau$.

such that close to $T_{\mathrm{M}}$ the AFM phase may lose its thermodynamic stability (also see Supplementary Fig. 6).

We note that the frequency difference between the excited phonon $(17 \mathrm{THz})$ and the magnon $(0.2 \mathrm{THz})$ rules out direct phonon-magnon coupling. To describe our experimental findings, we propose a simple phenomenological model, which indicates that all the changes of the magnetic potential can be explained by a renormalization of a single value that parametrizes the strength of the Fe-Dy exchange by about $5 \mu \mathrm{eV}$ per unit cell (Supplementary Note 3 and Supplementary Fig. 6). To identify the microscopic pathways that connect the light-driven optical phonons with the transient changes in the Fe-Dy exchange, we used density functional theory (DFT) calculations (Methods and Supplementary Note 5). The computations suggest the presence of a transient coherent lattice distortion in response to resonant pumping of the $B_{u}$ mode. This distortion has the same lifetime as the infrared-active mode $(\sim 1 \mathrm{ps})$ and emerges as a consequence of an anharmonic interaction of different phonon modes, generally known as ionic Raman scattering or nonlinear phononics ${ }^{28}$, which rectifies and transfers the large-amplitude excitation of the $B_{u}$ polar mode into a finite 

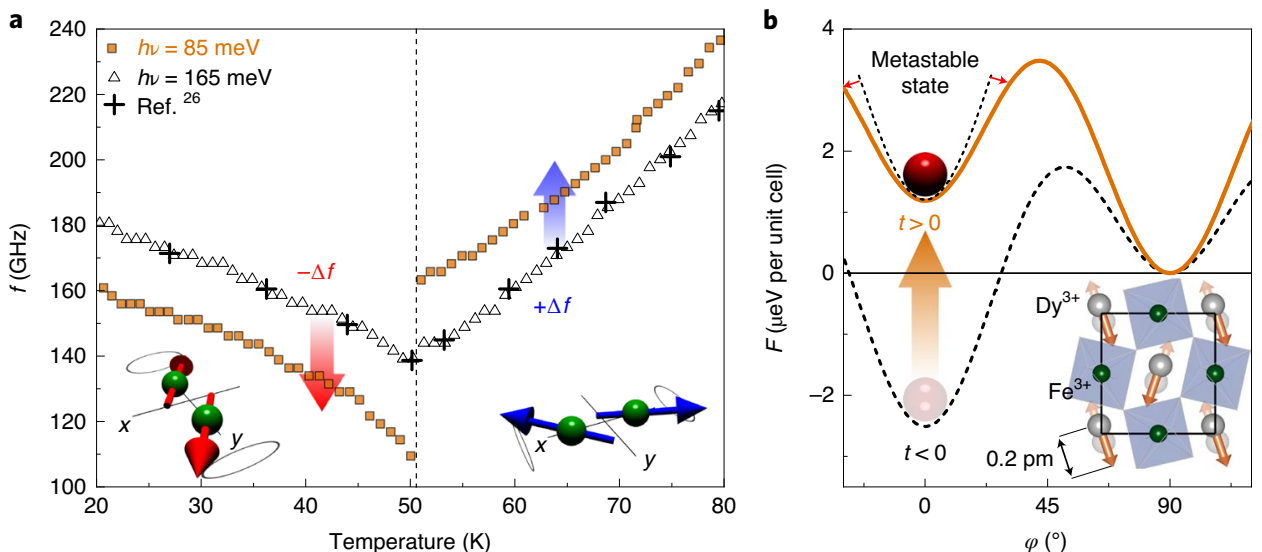

Fig. 3 | Out-of-equilibrium metastable magnetic state. a, Frequency of the spin precession as a function of the temperature across the Morin phase transition for different photon energies of the pump excitation. $\mathbf{b}$, Reconstructed magnetic potential $F$ before $(t<0)$ (dashed black line) and after ( $t>0$ ) (solid orange line) the phonon pumping. The position of the red ball represents the energy state of the system. Inset: the eigenmode of the $A_{g}$ lattice distortion, which is being considered as a driving force for the dynamics of the magnetic potential. The orange arrows depict the motions of the Dy ${ }^{3+}$ ions, antipolar in the adjacent layers.
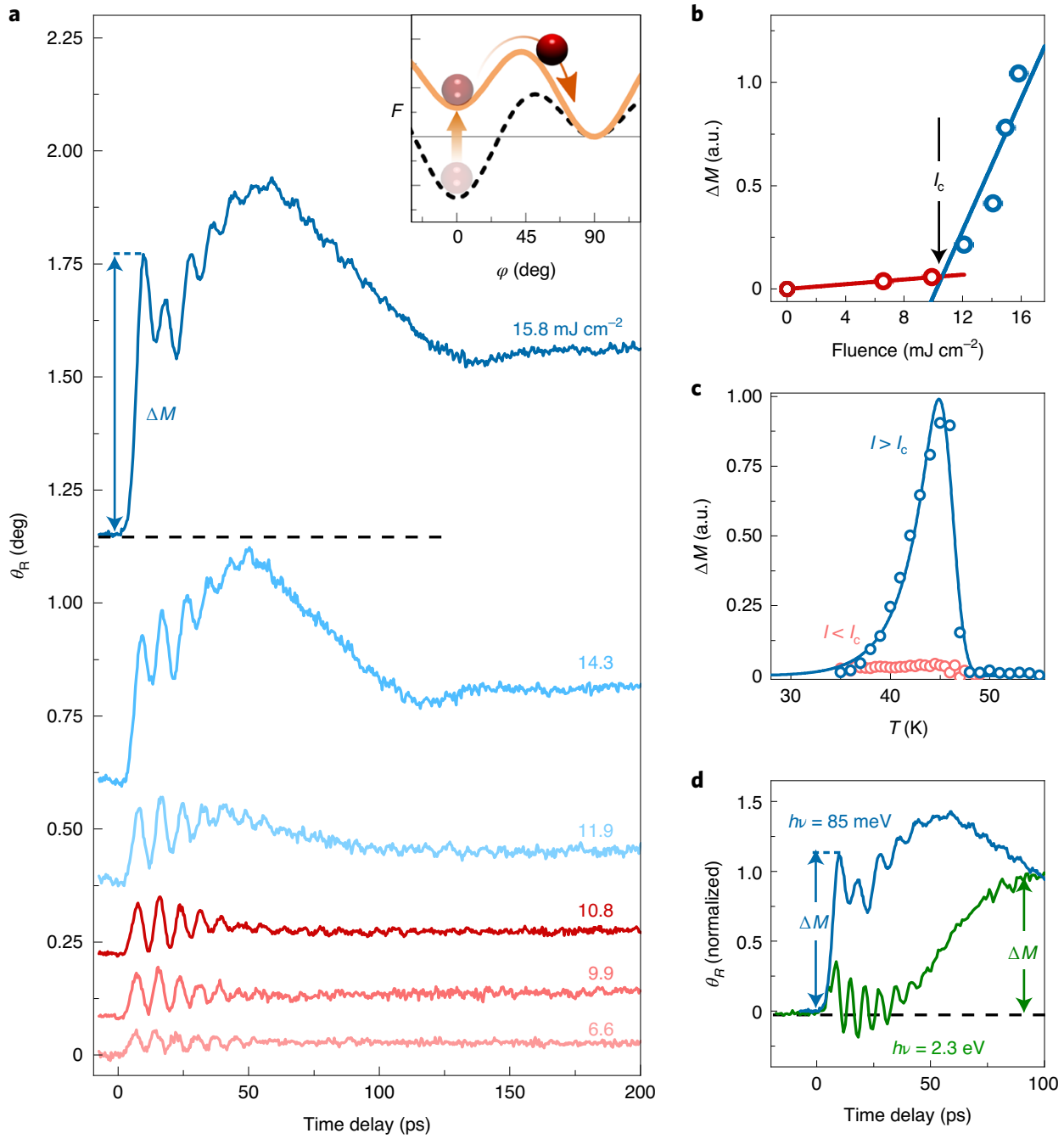

Fig. 4 | Ultrafast phonon-induced magnetic phase transition. a, Time-resolved dynamics of the polarization rotation $\theta_{R}$ at $T=45 \mathrm{~K}$ for various fluences of the pump. Each trace was extracted by subtracting individual traces obtained for opposite polarities of the external magnetic field to highlight the magnetic components of the dynamics. Inset: schematics of the ballistic reorientation in the altered magnetic potential $F$. $\mathbf{b}$, Amplitude of the transient magnetization $\Delta M$ as a function of the pump fluence I. c, $\Delta M$ as a function of the sample temperature for fluences $/$ above (blue curve) and below (red curve) the threshold $I_{\mathrm{c}}$. $\mathbf{d}, \mathrm{A}$ direct comparison of the dynamics of the magnetization initiated by a pump pulse with above-bandgap photon energy ( $2.3 \mathrm{eV}$ ) and in resonance with the phonon mode $(85 \mathrm{meV})$. 
time distortion along the coordinate of a coupled, Raman-active $\mathrm{A}_{\mathrm{g}}$ phonon mode (Fig. $3 \mathrm{~b}$ inset and Supplementary Fig. 9). This lattice distortion involves antipolar motions of the heavy $\mathrm{Dy}^{3+}$ ions reaching values of about $0.2 \mathrm{pm}$ for the pump fluences employed in our experiment (Supplementary Note 5 and Supplementary Fig. 10). The DFT results also show that such displacements can alter the Fe-Dy exchange integral by about $1-2 \mu \mathrm{eV}$ per unit cell, consistent with the estimation of the phenomenological model (Supplementary Fig. 11). The long-living effect of the distortion on the Fe-Dy exchange is presumably governed by the spin-lattice interaction, which also defines the lifetime of the spin precession (Fig. 2b inset).

To demonstrate the potential of the phonomagnetism for ultrafast control of magnetic states, we carried out phonomagnetic modifications of the RE-TM exchange interaction in proximity to $T_{\mathrm{M}}$. Two distinct regimes of the magnetization dynamics were observed above and below a critical fluence $I_{c}=10 \mathrm{~mJ} \mathrm{~cm}^{-2}$ (Fig. 4a,b). For fluences below $I_{c}$, the pump drives a nearly harmonic response of spins and mainly influences its amplitude and frequency. However, on exceeding the value of $I_{c}$, the course of the magnetization dynamics changes dramatically. A long-lived ( $>250 \mathrm{ps}$ ) offset, of odd parity with respect to the sign of the applied field, develops within nearly a half-period of the spin precession $(<5 \mathrm{ps})$, which marks the coherent emergence of a transient magnetization $\Delta M$. As the temperature is varied, it becomes apparent that the induced magnetization peaks nearly at $T_{\mathrm{M}}$ and completely vanishes for $T>T_{\mathrm{M}}$ (Fig. $4 \mathrm{c}$ ). The presence of a well-defined fluence threshold, as well as the observation of the phonon-induced macroscopic magnetization inherent to the WFM phase, are clear signatures that phonon pumping drives the coherent reorientation of spins accompanied by a change in the magnetic symmetry.

In Fig. 4d we compare the spin-reorientation transition obtained in $\mathrm{DyFeO}_{3}$ via conventional ultrafast heating (excitation with photon energy $2.3 \mathrm{eV}$ above the material's bandgap) with the phonomagnetism discussed here (also see Supplementary Note 6 and Supplementary Fig. 12). We note that ultrafast heating leads to the development of a net magnetization on a time scale of $100 \mathrm{ps}$, consistent with the spin-lattice relaxation time ${ }^{24}$. In contrast, the phonomagnetic route develops light-induced magnetism within a half cycle of the spin precession, below $5 \mathrm{ps}$, which indicates its coherent character.

By resonantly pumping infrared-active phonons with strong-field ultrashort multiterahertz pulses of light, we investigated a new route to reshape magnetic potentials and initiate ultrafast coherent magnetic phase transitions. Using a phenomenological description and first-principles calculations, we demonstrated that the mechanism is based on a phonon-driven change of the exchange interaction between the RE and TM ions. We anticipate that a similar mechanism is active in other magnetic materials that feature anisotropic exchange interactions, such as RE-based compounds (manganites, vanadates and orthochromites) and type II multiferroics ${ }^{29}$. Our findings fill the gap between the manipulation of magnetism with single-cycle terahertz pulses ${ }^{9,11}$ and with ultrashort pulses at optical frequencies ${ }^{6}$, thereby highlighting new avenues for the manipulation of ferroic order on ultrafast timescales and provides a new platform for magnonics and AFM spintronics ${ }^{30}$.

\section{Online content}

Any methods, additional references, Nature Research reporting summaries, source data, extended data, supplementary information, acknowledgements, peer review information; details of author contributions and competing interests; and statements of data and code availability are available at https://doi.org/10.1038/ s41563-021-00922-7.
Received: 21 May 2020; Accepted: 7 January 2021;

Published online: 8 February 2021

\section{References}

1. Fausti, D. et al. Light-induced superconductivity in a stripe-ordered cuprate. Science 331, 189-191 (2011).

2. Mankowsky, R. et al. Nonlinear lattice dynamics as a basis for enhanced superconductivity in $\mathrm{YBa}_{2} \mathrm{Cu}_{3} \mathrm{O}_{65}$. Nature 516, 71-73 (2014).

3. Mankowsky, R., von Hoegen, A., Först, M. \& Cavalleri, A. Ultrafast reversal of the ferroelectric polarization. Phys. Rev. Lett. 118, 197601 (2017).

4. Nova, T., Disa, A., Fechner, M. \& Cavalleri, A. Metastable ferroelectricity in optically strained $\mathrm{SrTiO}_{3}$. Science 364, 1075-1079 (2019).

5. Rini, M. et al. Control of the electronic phase of a manganite by mode-selective vibrational excitation. Nature 449, 72-74 (2007).

6. Kirilyuk, A., Kimel, A. V. \& Rasing, Th. Ultrafast optical manipulation of magnetic order. Rev. Mod. Phys. 82, 2731-2784 (2010).

7. Basov, D., Averitt, R. \& Hsieh, D. Towards properties on demand in quantum materials. Nat. Mater. 16, 1077-1088 (2017).

8. Stupakiewicz, A., Szerenos, K., Afanasiev, D., Kirilyuk, A. \& Kimel, A. Ultrafast nonthermal photo-magnetic recording in a transparent medium. Nature 542, 71-74 (2017).

9. Baierl, S. et al. Nonlinear spin control by terahertz-driven anisotropy fields. Nat. Photon. 10, 715-718 (2016).

10. Mikhaylovskiy, R. et al. Ultrafast optical modification of exchange interactions in iron oxides. Nat. Commun. 6, 8190 (2015).

11. Kampfrath, T. et al. Coherent terahertz control of antiferromagnetic spin waves. Nat. Photon. 5, 31-34 (2011).

12. Schlauderer, S. et al. Temporal and spectral fingerprints of ultrafast all-coherent spin switching. Nature 569, 383-387 (2019).

13. Först, M. et al. Melting of charge stripes in vibrationally driven $\mathrm{La}_{1.875} \mathrm{Ba}_{0.125} \mathrm{CuO}_{4}$ : assessing the respective roles of electronic and lattice order in frustrated superconductors. Phys. Rev. Lett. 112, 157002 (2014).

14. Tobey, R., Prabhakaran, D., Boothroyd, A. \& Cavalleri, A. Ultrafast electronic phase transition in $\mathrm{La}_{1 / 2} \mathrm{Sr}_{3 / 2} \mathrm{MnO}_{4}$ by coherent vibrational excitation: evidence for nonthermal melting of orbital order. Phys. Rev. Lett. 101, 197404 (2008).

15. Melnikov, A. et al. Coherent optical phonons and parametrically coupled magnons induced by femtosecond laser excitation of the $\mathrm{Gd}(0001)$ surface. Phys. Rev. Lett. 91, 227403 (2003).

16. Nova, T. F. et al. An effective magnetic field from optically driven phonons. Nat. Phys. 13, 132-136 (2017).

17. Maehrlein, S. F. et al. Dissecting spin-phonon equilibration in ferrimagnetic insulators by ultrafast lattice excitation. Sci. Adv. 4, eaar5164 (2018).

18. Disa, A. S. et al. Polarizing an antiferromagnet by optical engineering of the crystal field. Nat. Phys. 16, 937-941 (2020).

19. Zvezdin, A. \& Matveev, V. Theory of the magnetic properties of dysprosium orthoferrite. Sov. Phys. JETP 50, 543-548 (1979).

20. Khim, T.-Y. et al. Strain control spin reorientation transition in $\mathrm{DyFeO}_{3} /$ $\mathrm{SrTiO}_{3}$ epitaxial film. Appl. Phys. Lett. 99, 072501 (2011).

21. Fechner, M. et al. Magnetophononics: ultrafast spin control through the lattice. Phys. Rev. Mater. 2, 064401 (2018).

22. Juraschek, D. M., Narang, P. \& Spaldin, N. A. Phono-magnetic analogs to opto-magnetic effects. Phys. Rev. Res. 2, 043035 (2020).

23. Hase, M., Kitajima, M., Nakashima, S.-i \& Mizoguchi, K. Dynamics of coherent anharmonic phonons in bismuth using high density photoexcitation. Phys. Rev. Lett. 88, 067401 (2002).

24. Yamaguchi, K., Kurihara, T., Watanabe, H., Nakajima, M. \& Suemoto, T. Dynamics of photoinduced change of magnetoanisotropy parameter in orthoferrites probed with terahertz excited coherent spin precession. Phys. Rev. B 92, 064404 (2015).

25. Kalashnikova, A. et al. Impulsive generation of coherent magnons by linearly polarized light in the easy-plane antiferromagnet $\mathrm{FeBO}_{3}$. Phys. Rev. Lett. 99, 167205 (2007).

26. Balbashov, A., Volkov, A., Lebedev, S., Mukhin, A. \& Prokhorov, A. High-frequency magnetic properties of dysprosium orthoferrite. Zh. Eksp. Teor. Fiz. 88, 974-987 (1985).

27. Berton, A. \& Sharon, B. Specific heat of $\mathrm{DyFeO}_{3}$ from $1.2^{\circ}-80^{\circ}$ K. J. Appl. Phys. 39, 1367-1368 (1968).

28. Subedi, A., Cavalleri, A. \& Georges, A. Theory of nonlinear phononics for coherent light control of solids. Phys. Rev. B 89, 220301 (2014).

29. Tokura, Y., Seki, S. \& Nagaosa, N. Multiferroics of spin origin. Rep. Prog. Phys. 77, 076501 (2014).

30. Baltz, V. et al. Antiferromagnetic spintronics. Rev. Mod. Phys. 90, 015005 (2018).

Publisher's note Springer Nature remains neutral with regard to jurisdictional claims in published maps and institutional affiliations.

๑) The Author(s), under exclusive licence to Springer Nature Limited 2021 


\section{Methods}

Sample and experimental set-up. We used a monocrystalline, $63-\mu \mathrm{m}$-thick $\mathrm{DyFeO}_{3}$ sample grown by floating-zone melting. The sample was cut perpendicularly to the $z$ crystallographic axis in the form of a thin slab. The sample was kept in a dry-cycle cryostat (Montana Instruments), which allowed it to cool down to $10 \mathrm{~K}$, far below $T_{\mathrm{M}}$. Static bias fields up to $700 \mathrm{mT}$ from an electromagnet were applied within the $x z$ plane of the crystal at an angle of about $70^{\circ}$ relative to the $z$ axis to provide a single domain magnetic state of the sample.

The intense pump pulses with wavelengths in the MIR spectral range used in this experiment were generated via difference frequency generation in a GaSe crystal using the output beams of two commercially available, independently tunable optical parametric amplifiers (OPAs) integrated into a single housing (Light Conversion, TOPAS-Twins). The OPAs were pumped by a commercially available amplified Ti:sapphire laser system (Coherent, Astrella), which delivered pulses at a $1 \mathrm{kHz}$ repetition rate with a duration of $100 \mathrm{fs}$ and a photon energy of $1.55 \mathrm{eV}(\lambda=0.8 \mu \mathrm{m})$. The OPAs were seeded by the same white light generated in a sapphire crystal, which ensures separately tunable, but phase-locked output pulses $^{31}$ with photon energies in the range $0.45-1.1 \mathrm{eV}(\lambda=2.7-1.1 \mu \mathrm{m})$. As a result, when the pulses were mixed in the GaSe crystal, the generated MIR pulses were carrier envelope phase stable ${ }^{32}$, linearly polarized and their energy lay in the range $65-250 \mathrm{meV}(\lambda=19-5 \mu \mathrm{m})$ with an average pulse duration of around $250 \mathrm{fs}$. The scheme of the set-up for generation of the MIR pulses (MIR converter) is shown in Supplementary Fig. 1.

To generate pump pulses in the visible spectrum with a photon energy larger than that of the electronic bandgap, we used a $\beta$-barium borate single crystal to double or triple the photon energy of a single OPA output. This provided tunable excitation pulses with energies in the range 0.9-3.3 eV (Supplementary Fig. 1).

In the experiments, the mid-infrared pulses were focused onto the sample surface to a spot with a diameter of about $150 \mu \mathrm{m}$ using an off-axis parabolic mirror. The pump-induced dynamics were measured in a transmission geometry. The polarization rotation $\theta_{\mathrm{R}}$ of the linearly polarized probe pulse, imprinted by the magneto-optical Faraday effect, was measured with the help of a polarization bridge (Wollaston prism) and a pair of balanced Si photodetectors.

The loss function of the sample in the MIR spectral range was measured using a Nicolet 6700 Fourier transform infrared spectrometer. The optical absorption in the visible spectral range was measured using a halogen lamp and an Ocean Optics spectrometer.

DFT calculations. We simulated the Pnma phase of $\mathrm{DyFeO}_{3}$ through $\mathrm{DFT}^{33,34}$ and its projected augmented wave (PAW) $)^{35}$ flavour as implemented in the ABINIT package $^{36-38}$. The PAW atomic potentials used for Fe and $\mathrm{O}$ were downloaded from the GBRV (Garrity-Bennett-Rabe-Vanderbilt) pseudopotential library ${ }^{39}$ with $3 s$, $3 p, 3 d$ and $4 s$, and $2 s$ and $2 p$ considered as valence states for Fe and $\mathrm{O}$, respectively. For Dy, we used the PAW atomic potential from Topsakal and Wentzcovitch ${ }^{40}$ in which the valence states are $4 f, 5 s, 5 p$ and $6 s$ (with $f$ electrons in the valence). We used the PBEsol GGA functional ${ }^{41}$ for the exchange correlation interaction and Hubbard corrections ${ }^{42}$ on both Fe and Dy of, respectively, 4 and $7 \mathrm{eV}$, to have the closest possible properties with respect to the experiments (lattice constants). All the calculations were done with a $6 \times 6 \times 4$ mesh of $k$ points for the reciprocal space and a cutoff energy on the plane-wave expansion of 36 Hartree and 72 Hartree for the second finer grid inside the PAW spheres. To calculate the phonons, the frozen phonon technique was used through the phonopy software ${ }^{43}$.

\section{Data availability}

All data presented in this work are publicly available with identifier https://doi.

org/10.5281/zenodo.4338556. Source data are provided with this paper.

\section{Code availability}

The TB2J code for calculating the exchange interactions is freely available under the BSD 2 clause license and can be found at https://github.com/mailhexu/TB2J/. The Abinit code for the DFT calculations is an open source code with GNU General Public License and is freely available at https://www.abinit.org/.

\section{References}

31. Sell, A., Leitenstorfer, A. \& Huber, R. Phase-locked generation and field-resolved detection of widely tunable terahertz pulses with amplitudes exceeding $100 \mathrm{MV} / \mathrm{cm}$. Opt. Lett. 33, 2767-2769 (2008).
32. Baltuška, A., Fuji, T. \& Kobayashi, T. Controlling the carrier-envelope phase of ultrashort light pulses with optical parametric amplifiers. Phys. Rev. Lett. 88, 133901 (2002).

33. Hohenberg, P. \& Kohn, W. Inhomogeneous electron gas. Phys. Rev. 136, B864 (1964).

34. Kohn, W. \& Sham, L. J. Self-consistent equations including exchange and correlation effects. Phys. Rev. 140, A1133 (1965)

35. Blöchl, P. E. Projector augmented-wave method. Phys. Rev. B 50, 17953 (1994).

36. Gonze, X. et al. First-principles computation of material properties: the ABINIT software project. Comput. Mater. Sci. 25, 478-492 (2002).

37. Gonze, X. et al. Recent developments in the ABINIT software package. Comput. Phys. Commun. 205, 106-131 (2016).

38. Torrent, M., Jollet, F., Bottin, F., Zérah, G. \& Gonze, X. Implementation of the projector augmented-wave method in the ABINIT code: application to the study of iron under pressure. Comput. Mater. Sci. 42, 337-351 (2008).

39. Garrity, K. F., Bennett, J. W., Rabe, K. M. \& Vanderbilt, D. Pseudopotentials for high-throughput DFT calculations. Comput. Mater. Sci. 81, 446-452 (2014)

40. Topsakal, M. \& Wentzcovitch, R. Accurate projected augmented wave (PAW) datasets for rare-earth elements $(\mathrm{RE}=\mathrm{La}-\mathrm{Lu})$. Comput. Mater. Sci. 95 , 263-270 (2014).

41. Perdew, J. P. et al. Restoring the density-gradient expansion for exchange in solids and surfaces. Phys. Rev. Lett. 100, 136406 (2008).

42. Liechtenstein, A., Anisimov, V. I. \& Zaanen, J. Density-functional theory and strong interactions: orbital ordering in Mott-Hubbard insulators. Phys. Rev. B 52, R5467 (1995).

43. Togo, A. \& Tanaka, I. First principles phonon calculations in materials science. Scr. Mater. 108, 1-5 (2015).

\section{Acknowledgements}

We thank S. Heirman for assistance in measuring the linear absorption in the MIR spectral range, E. Lesne for the X-ray diffraction measurements and E. Demler, R. Citro, M. Cuoco and T. C. van Thiel for fruitful discussions. This work was supported by the EU through the European Research Council, grant no. 677458 (AlterMateria), the Netherlands Organization for Scientific Research (NWO/OCW) as part of the Frontiers of Nanoscience programme (NanoFront) and the VENI-VIDI-VICI programme, the European Union's Seventh Framework Programme (FP7/2007-2013)/ERC grant agreement no. 339813 (Exchange), ERC Grant agreement 852050 (MAGSHAKE), the programme Leading Scientist of the Russian Ministry of Science and Higher Education (14.Z50.31.0034), the Ministry of Science and Higher Education of the Russian Federation in the framework of Increase Competitiveness Program of NUST MISiS (grant no. K2-2019-006), implemented by a governmental decree dated 16 March 2013, N211. E.B. and A.S. thank the FRS-FNRS, ARC AIMED project, the CECI supercomputer facilities (grant no. 2.5020.1) and Tier-1 supercomputer of the Fédération Wallonie-Bruxelles funded by the Walloon Region (grant no. 1117545)

\section{Author contributions}

A.D.C. conceived the project together with D.A. D.A. and J.R.H. carried out the experiments and analysed the data. R.V.M. and A.V.K. identified the material system for the project and contributed to the analysis. B.A.I. and Y.M.B. contributed to the theoretical treatment of the experimental results. A.S. and E.B. performed the DFT calculations. All the authors discussed the results. The manuscript was written by D.A. J.R.H. and A.D.C. with feedback from all the co-authors.

\section{Competing interests}

Authors declare no competing interests.

\section{Additional information}

Supplementary information The online version contains supplementary material available at https://doi.org/10.1038/s41563-021-00922-7.

Correspondence and requests for materials should be addressed to D.A. or A.D.C.

Peer review information Nature Materials thanks the anonymous reviewers for their contribution to the peer review of this work.

Reprints and permissions information is available at www.nature.com/reprints. 